\title{
The history and scope of neuropsychiatry
}

\author{
Michael Trimble*
}

Institute of Neurology, London, UK

Received 30 October 2017; Accepted 16 November 2017; First published online 16 May 2018

This is the second special edition of CNS Spectrums that addresses topics of neurospychiatry-one of the initial aims of the journal. In that earlier edition, the background history of the discipline we now recognize as neuropsychiatry-allied to but distinct from both neurology and psychiatry-was presented. Central were the neuroanatomical developments outlining areas of the brain primarily linked with feelings and expression of emotion with previously unappreciated widespread connections to cortical areas, providing the substrate for an understanding of the complexity of clinical neuropsychiatric presentations. From a neurophysiological perspective, the EEG and the discovery of temporal lobe epilepsy, and the co-morbid psychopathological associations linked with epilepsy and movement disorders opened clinical avenues for modern neuropsychiatry. Conceptually there has been the shift from the empiricist view of the brain as a passive receptacle of sensory impressions to one in which the brain is seen as an active, seeking, creative organ, the intentional brain, with emotions embodied and directing motion (e-motion), but also cognitions and rationality. ${ }^{1}$

With stark divisions between neurology and psychiatry, the failure of the older framework to include the growing number of patients presenting with emotional and behavioral consequences of brain dysfunction insisted on a need for practitioners with a brain-based view of behavioral disorders. This must be allied with the broad dimensions of human cultural and social factors leading to the signs, the symptoms, and the progression of illness, the latter being distinct from disease. In the earlier edition were articles that covered non-epileptic seizures, the comorbid psychiatric disorders of epilepsy, with a separate article on the psychoses of epilepsy, and a brief history of neuropsychiatry from the Greeks to the 20th century. Modern neuropsychiatry was presented as an important discipline of today's clinical neuroscience.

\footnotetext{
* Address for correspondence: Michael Trimble, Institute of Neurology, Queen Square, London WC1N3BG, UK.

(Email: mtrimble@ion.ucl.ac.uk)
}

In this second edition, important neuropsychiatric investigations and some other disorders are discussed. The neuropsychiatric examination requires a certain expertise of vision and touch, which are not a part of either a routine neurological or psychiatric evaluation, yet subtle clues offer up diagnostic possibilities, varied for the patient under observation. The key points are outlined by Sheldon Benjamin and Margo Lauterbach, and are intended to enhance our clinical expertise, noting parts of the examination not taught routinely in neurology or psychiatry.

There is a review of the use of electroencephalography (EEG) by Nash Boutros. It was the availability of the EEG in the second half of the 20th century that firmly set modern neuropsychiatry within a new framework of the clinical domains of psychiatry and neurology. But the techniques and allied developments also aided the development of psychopharmacology, and revived interest in mind-brain relationships. Electrophysiological techniques are quite underutilized in current neuropsychiatry, but are often used inappropriately, leading many patients with for example non-epileptic seizures being diagnosed as having epilepsy and mismanaged on account of misinterpretation of their recordings. There is an article on the Giles de la Tourette syndrome from Andrea Cavanna in Birmingham, England, whose team has special interest in the syndrome. Once considered only a "tic" disorder, hence a motor aberration, the rich spectrum of comorbidities and the neurophysiological and brain imaging findings render this a model neuropsychiatric disorder. There is a review of atypical Alzheimer's disease by Bradford Dickerson and his colleagues. This emphasises that the clinical spectrum of the dementias requires careful evaluation, with different shades of cognitive and behavioral patterns and management needs depending on the underlying pathology and distribution of cerebral changes. Finally included is an article related to the assessment and management of functional neurological (conversion) disorders from an occupational therapist (OT) perspective from John Stone, Alan Carson, and his group from Edinburgh who have done such important 
work taking up the challenge of this patient population with an enthusiasm not published since perhaps the treatise of Paul Briquet. The diagnosis is still one of controversy and confusion, but it is more frequently made as newer techniques of investigation and greater clinical sophistication are nuanced and multidisciplinary management programs established. ${ }^{2}$

It is hoped that these two editions of CNS Spectrums, with concern to bring modern neuropsychiatry to a wider audience, will encourage those in training to consider the specialty as a career option, and help guide practitioners toward further research and clinical innovation in one of the most fascinating specialties in medicine.

\section{Disclosures}

Professor Trimble has nothing to disclose.

REFERENCES:

1. Trimble MR. The Intentional Brain: Motion, Emotion and the Development of Modern Neuropsychiatry. Baltimore, MD: Johns Hopkins Press; 2016.

2. Hallet M, Stone J, Carson AJ, eds. Functional Neurologic Disorders. London: Elsevier; 2016. Handbook of Clinical Neurology; vol 139. 